\title{
Targeting epigenetic mechanisms in Parkinson's disease: HDAC1 inhibitor LG325 modulates circuitry imbalance
}

\author{
Catarina Cunha ${ }^{1,2^{*}}$, Maria Novella Romanelli ${ }^{3}$ \\ ${ }^{1}$ Department of Child and Adolescent Psychiatry, New York University Grossman School of Medicine, \\ New York, NY 10016 \\ ${ }^{2}$ Emotional Brain Institute, Nathan Kline Institute, Orangeburg, NY, USA \\ ${ }^{3}$ Department of Neurosciences, Psychology, Drug Research and Child Health (NEUROFARBA), Section \\ of Pharmaceutical and Nutraceutical Sciences, University of Florence, Via Ugo Schiff 6, 50019 Sesto \\ Fiorentino, Italy \\ ${ }^{*}$ Correspondence: Catarina Cunha, Emotional Brain Institute, Nathan Kline Institute, Orangeburg, NY, \\ USA, E-mail: catarina.cunha@nki.rfmh.org
}

\begin{abstract}
Parkinson's disease (PD) is a prevalent neurodegenerative disorder worldwide that affects the US alone ca. 1 million people. There is limited evidence for genetic associations with PD; however, recent research proposes epigenetics as a mechanism involved in PD's development and progression. Especially histone deacetylase (HDAC) inhibitors were shown to recuperate synaptic plasticity, learning, and memory in several neurodegenerative disorders. Therefore, we studied the effects of LG325 (specific HDAC1 inhibitor) on striatal spiny projection neurons (SPNs), which are known to be involved in PD motor dysfunctions, targeting direct pathway SPNs (dSPNs) and indirect pathway SPNs (iSPNs) separately by performing 2-photon laser scanning microscopy in BAC transgenic drd1a-tdTomato and drd2-GFP transgenic mice. We evoked single backpropagating action potentials (bAP) that cause a somewhat higher elevation in cytosolic Ca2+ concentration at distal dendritic locations in iSPNs compared to dSPNs. Bath application of $1 \mu \mathrm{M}$ LG325 lead to a marked decrease in the bAPs evoked dendritic Ca2+ entry in dSPNs. We propose that HDAC1 inhibition could possibly not only alleviate the progression of PD but may also hold the potential to relieve acutely motor symptoms in PD patients. HDAC1 inhibitors should be further investigated and considered a treatment option, especially in patients who have reached a resistance to dopamine-related treatments.
\end{abstract}


Parkinson's disease (PD) is one of the most common neurodegenerative disorders, and reports estimate that approximately 1 million Americans currently suffer from this illness ${ }^{1}$. Only limited data support the genetic associations with PD ${ }^{2}$. However, numerous risk factors are being associated with PD development, which consists of oxidative stress, the building up of free radicals, and toxins ${ }^{3,4}$. Studies showed a surprising counter correlation connecting cigarette smoking, caffeine intake, and the probability of PD ${ }^{5}$. Dr. James Parkinson in 1817 was the first person to describe PD as a "shaking palsy." PD is a chronic, progressive neurodegenerative illness that affects motor and non-motor functions. PD is a symptom complex, which includes resting tremor, bradykinesia, and muscular rigidity. In the 1950s, researchers identified striatal dopamine depletion as the primary cause of PD's motor symptoms. The accumulation of Lewy bodies (LBs) are proposed to be the underlying cause of the progressive degeneration of dopaminergic neurons in the substantia nigra pars compacta $(\mathrm{SNpc})$, which project to the striatum (the nigrostriatal pathway) ${ }^{6-10}$. However, patients show motor deficiencies only after losing $50 \%-80 \%$ of dopaminergic neurons. This suggests that a compensatory mechanism in the early stages of the disease must be holding up the symptom development of PD ${ }^{11}$. PD treatments aim to restore dopamine activity in the striatum through D2 and D1 receptor activation ${ }^{12}$. Dopaminergic medications improve the motor symptoms and quality of life of PD patients only in the early stages. Usually, after a few years of dopaminergic treatments, PD patients develop a "dopa-resistance, "which lead to motor symptoms such as speech impairment, abnormal posture, gait, and balance problems, and "dopa-resistant" non-motor signs, which consist of autonomic dysfunction, mood, and cognitive impairment, sleep problems, pain and also drug-induced side effects being psychosis, motor fluctuations, and dyskinesia $4,13,14$. Many clinicians conclude that the current PD treatments hold room for improvement concerning efficacy and safety ${ }^{15}$.

There are two types of dopamine (DA) receptors, D1 (excitatory) and D2 (inhibitory), that modulate motor activity in the extrapyramidal system. This system consists of the basal ganglia, which includes the internal globus pallidal segment (GPi) of the striatum, and the pars reticulata of the substantia nigra ( $\mathrm{SNpr}$ ), which then influences larger circuits located in the thalamus and the cortex. In summary, the depletion of dopamine in the striatum in $\mathrm{PD}$ results in higher activity of the GPi/SNpr circuits, resulting in inhibition of the thalamus 
and decreased activation of the frontal cortex of the thalamus, which leads to decreased motor activity in PD 16-19. In more detail, the current model consists of the "direct" and "indirect" pathway ("go" or "no-go" pathway), which proposes that dopamine depletion changes the activity of spiny projection neurons (SPNs) in the striatum, which leads to an imbalance in the activity of the direct pathway SPNs (dSPNs) and indirect pathway SPNs (iSPNs). The SPNs represent the striatal output, so this imbalance is proposed to have marked consequences for motor movements and behavior 20,21. A study performed in acute brain slices showed that DA depletion leads to enhanced back-propagating action potentials (bAP)-evoked $\mathrm{Ca} 2+$ transients in distal dendrites and spines of the iSPNs, which was proposed to be an underlying mechanism potentially contributing to adaptations in PD models ${ }^{22}$.

Recent research suggests that epigenetics also play a significant role in the development and progression of PD 23-27. Epigenetic mechanisms are defined as modulations in gene expression without changes in the sequence of the DNA. The mechanisms consist mainly of DNA methylation, post-modifications of histones, and non-coding RNAs ${ }^{28}$. These epigenetic mechanisms have been suggested as promising targets for future diagnostic and therapeutic strategies.

Histone acetylation and deacetylation are epigenetic mechanisms that play a significant role in maintaining chromatin stability and learning and memory. Recent studies have shown that HDAC inhibitors improve synaptic plasticity, learning, and memory in various neurodegenerative disorders. More specifically, the use of histone deacetylase (HDAC1) inhibiting agents have been hypothesized to mitigate the pathological imbalance observed in PD ${ }^{29}$. Until this day, nobody has studied the effects of HDAC inhibitors on the striatal circuitry. LG325 has been shown to be an HDAC1 inhibitor that successfully ameliorated neuropathic pain in a mouse model, with very low side effects and toxicity ${ }^{30}$. We, therefore, studied the impact of LG325 on dSPNs and iSPNs in the striatal circuitry. We performed 2-photon laser scanning microscopy in acute brain slices of BAC transgenic drd1a-tdTomato and drd2-GFP transgenic mice while patch-clamping SPNs of the striatum. Single back-propagating action potentials (bAP) were evoked, which induced slightly higher elevations in cytosolic $\mathrm{Ca} 2+$ concentration at distal dendritic locations in iSPNs compared to dSPNs. The 30 min bath application of $1 \mu \mathrm{M}$ LG325 
decreased the dendritic Ca2+ entry elicited by bAPs only in dSPNs significantly. This result suggests that HDAC1 inhibition would possibly not just ameliorate PD progression but could also improve acute motor symptoms. Therefore, HDAC1 inhibitors should be further studied as a treatment option, especially for patients that have reached the "doparesistant" phase.

Figure 1
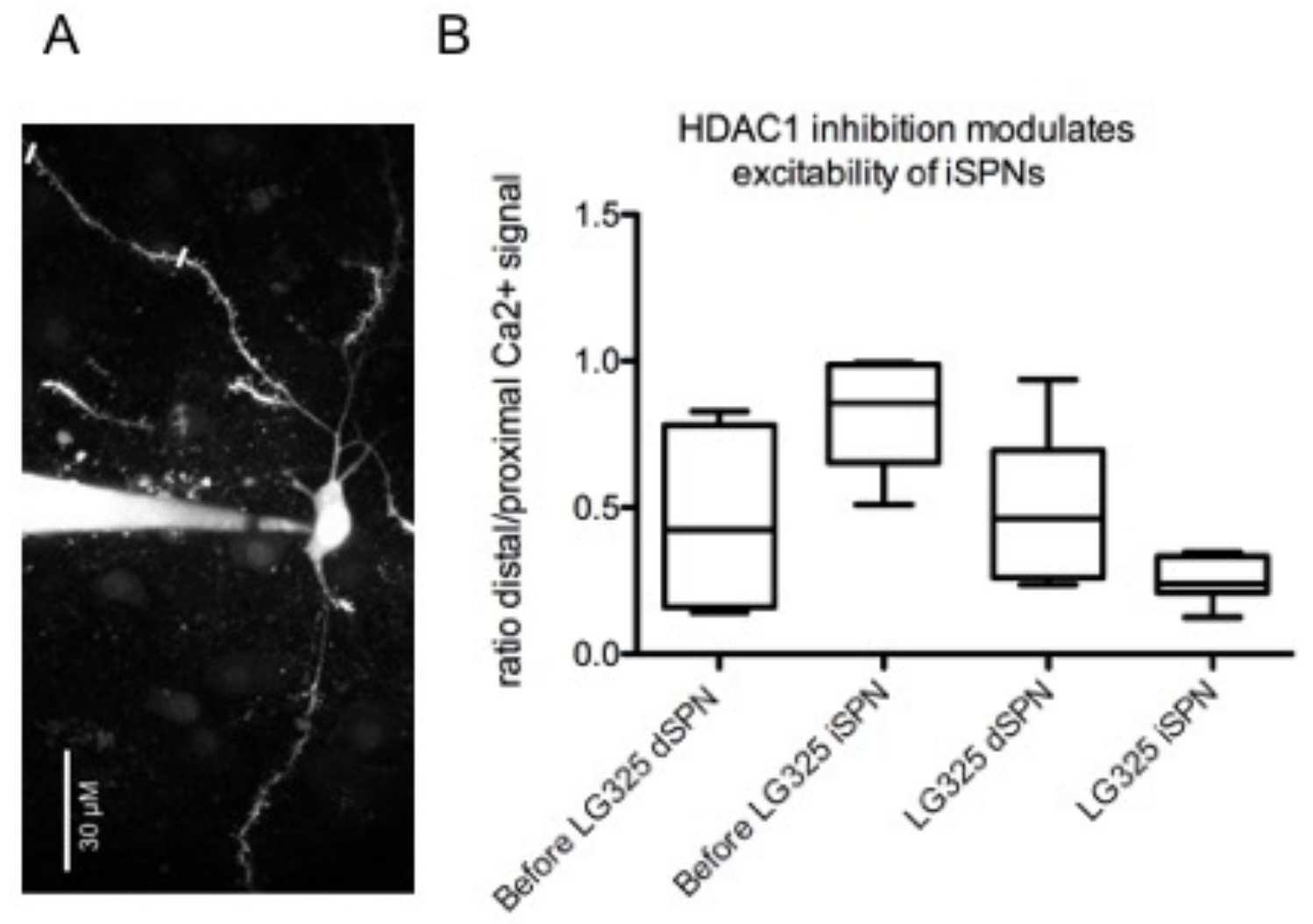

Fig. 1: HDAC1 inhibitor LG325 decreases excitability in iSPNs

(A) Image of a iSPN soma and dendrite from a BAC transgenic drd2-GFP transgenic mouse. We recorded the bAP-evoked $\mathrm{Ca} 2+$ transient at two different locations along each cell's dendrite $(60,100 \mu \mathrm{m})$. (B) Diagram summarizes the amplitudes of the bAP-evoked $\mathrm{Ca} 2+$ transient normalized to the proximal recording, comparing dSPNs to iSPNs $(n=5-$ 6 in each group; ANOVA, $p<0.05$ ).

\section{Methods}


Animals

For this study, 3-8-month-old male and female BAC transgenic mice were used expressing drd1a-tdTomato. The mice were singly housed with a $12 \mathrm{~h}$ light/dark cycle and food and water ad libitum. The study complied with the National Institutes of Health Guide for Care and Use of Laboratory Animals and was approved by the Animal Welfare Committee, NY, USA.

\section{Brain Slice Preparation}

We prepared para-sagittal brain slices $(275 \mu \mathrm{m})$ from 17-25-day BAC transgenic drd1atdTomato transgenic mice while anesthetized with a mixture of ketamine (50 mg kg-1) and xylazine (4.5 mg kg-1). After brain removal, it was dissected in oxygenated, ice-cold, artificial cerebral spinal fluid (ACSF (in mM): $124 \mathrm{NaCl}, 3 \mathrm{KCl}, 2 \mathrm{CaCl} 2,1 \mathrm{MgCl} 2,26$ NaHCO3, $1 \mathrm{NaH} 2 \mathrm{PO} 4$, and $10 \mathrm{D}-(+)$-glucose; $300 \mathrm{mOsm} / \mathrm{l})$ with a Leica VT1000S vibratome (Leica Microsystems, Wetzlar, Germany). The incubation time of the acute brain slices in ACSF was at $35^{\circ} \mathrm{C}$ for $45 \mathrm{~min}$. Whole-cell recordings were performed at room temperature, while ACSF solutions were bubbled with 95\%O2/5\%CO2 for oxygenation and a $\mathrm{pH} \approx 7.4$ maintenance.

2-photon laser scanning microscopy (2PLSM) ${ }^{22}$

The categorization of dSPNs and iSPNs was done by identifying tdTomato by using a Laser Scanning Microscope system using an $810 \mathrm{~nm}$ excitation. Internal solution consisted of (in mM): $135 \mathrm{KMeSO} 4,5 \mathrm{KCl}, 10 \mathrm{HEPES}, 2 \mathrm{MgATP}, 0.2 \mathrm{Na}$ GTP, 10 phosphocreatine, Alexa 568 and 0.5 Fluo-4F hydrazide $\mathrm{Na}+$ salt (Invitrogen), $\mathrm{pH}=7.25-$ 7.3 with $\mathrm{KOH}, 270 \mathrm{mOsm} / \mathrm{l}$. The recording electrodes were used with a resistance of $\sim 4$ $\mathrm{M} \Omega$.

\section{Electrophysiology and $\mathrm{Ca} 2+$ imaging}

The electrophysiological experiments were performed in whole-cell current-clamp settings with a Multiclamp 700B amplifier (Axon Instruments, Union City, CA), while single bAPs were elicited through current pulse injections (2 nA, 2 ms) at 5-second intervals. In prior recordings, the SPNs were filled with the Ca2+ indicator Fluo-4 (200 $\mu \mathrm{M})$ to perform 
2PLSM imaging of $\mathrm{Ca} 2+$ signals. The line-scan signals were recorded with a rate of $6 \mathrm{~ms}$ and 512 pixels per line with $0.078 \mu \mathrm{m}$ pixels and $10 \mu \mathrm{s}$ pixel dwell time and were initiated at $200 \mathrm{~ms}$ before stimulation and extended until four seconds after stimulation to establish background fluorescence and decay of signal after stimulation. The drug was bath applied for 30 min before the experiments.

Drug

LG325 (N1-Hydroxy-N6-(1-methyl-2-oxo-5-phenyl-2,3-dihydro-1Hbenzo[e][1,4] diazepine-3-yl)hexanediamide) was synthesized in the laboratory of Prof. Maria Novella Romanelli (University of Florence, Italy).

Data analysis

The data presented in this study consists of a spine, dendrite, or spine-dendrite pair recorded from a single SPN. Data were analyzed with IGOR Pro (WaveMetrics, Lake Oswego, OR). The data is presented as a mean value of the fluorescent signal over time $(F(t)$ consisting of 5 pixels. The baseline of fluorescence is presented as Fo, which consists of an average of our line scans' first 30 time points. We normalized the Ca2+ signal $(\triangle F / F o)$ with the basal fluorescence. We used IGOR to smooth the traces with a binomial Gaussian filter.

The statistical analysis was performed with GraphPad Prism 5.0b I which we performed one-way or two-way ANOVA with Tukey post hoc comparison test, and the statistical significance was defined as $p<0.05$.

Author contributions statement

Catarina Cunha conceived of the presented idea. Catarina Cunha developed the experiment design, performed experiments, and analyzed the data. Prof. Maria Novella Romanelli synthesized the HDAC inhibitor drug. All authors discussed the results and contributed to the final manuscript.

Competing interests statement 
All authors declare that they have no significant competing financial, professional, or personal interests that might have influenced the work's performance or presentation described in this manuscript.

Data availability statement

The data that support the findings of this study are available from the corresponding author (C.C.) upon reasonable request.

1 Marras, C. et al. Prevalence of Parkinson's disease across North America. NPJ Parkinsons Dis 4, 21, doi:10.1038/s41531-018-0058-0 (2018).

2 Spatola, M. \& Wider, C. Genetics of Parkinson's disease: the yield. Parkinsonism Relat Disord 20 Suppl 1, S35-38, doi:10.1016/S1353-8020(13)70011-7 (2014).

3 McCormack, A. L. et al. Environmental risk factors and Parkinson's disease: selective degeneration of nigral dopaminergic neurons caused by the herbicide paraquat. Neurobiol Dis 10, 119-127 (2002).

4 DeMaagd, G. \& Philip, A. Parkinson's Disease and Its Management: Part 1: Disease Entity, Risk Factors, Pathophysiology, Clinical Presentation, and Diagnosis. $P$ \& $T$ : a peerreviewed journal for formulary management 40, 504-532 (2015).

5 Liu, R. et al. Caffeine intake, smoking, and risk of Parkinson disease in men and women. Am J Epidemiol 175, 1200-1207, doi:10.1093/aje/kwr451 (2012).

6 Chaudhuri, K. R. \& Jenner, P. Two hundred years since James Parkinson's essay on the shaking palsy-Have we made progress? Insights from the James Parkinson's 200 years course held in London, March 2017. Mov Disord 32, 1311-1315, doi:10.1002/mds.27104 (2017).

$7 \quad$ Hurwitz, B. The Status of "Nonmotor" Features of the Malady in an Essay on the Shaking Palsy (1817). Int Rev Neurobiol 133, 3-12, doi:10.1016/bs.irn.2017.04.008 (2017).

8 Donaldson, I. M. James Parkinson's essay on the shaking palsy. J R Coll Physicians Edinb 45, 84-86, doi:10.4997/JRCPE.2015.118 (2015).

9 Kovari, E., Horvath, J. \& Bouras, C. Neuropathology of Lewy body disorders. Brain Res Bull 80, 203-210, doi:10.1016/j.brainresbull.2009.06.018 (2009).

10 McKeith, I., Fairbairn, A., Perry, R., Thompson, P. \& Perry, E. Neuroleptic sensitivity in patients with senile dementia of Lewy body type. BMJ 305, 673-678 (1992).

11 Blesa, J. et al. Compensatory mechanisms in Parkinson's disease: Circuits adaptations and role in disease modification. Exp Neurol 298, 148-161, doi:10.1016/j.expneurol.2017.10.002 (2017).

12 Beaulieu, J. M. \& Gainetdinov, R. R. The physiology, signaling, and pharmacology of dopamine receptors. Pharmacol Rev 63, 182-217, doi:10.1124/pr.110.002642 (2011). 
13 Sgroi, S. \& Tonini, R. Opioidergic Modulation of Striatal Circuits, Implications in Parkinson's Disease and Levodopa Induced Dyskinesia. Front Neurol 9, 524, doi:10.3389/fneur.2018.00524 (2018).

14 Barbin, L. et al. Non-homogeneous effect of levodopa on inhibitory circuits in Parkinson's disease and dyskinesia. Parkinsonism Relat Disord 19, 165-170, doi:10.1016/j.parkreldis.2012.08.012 (2013).

15 Rascol, O. et al. Limitations of current Parkinson's disease therapy. Ann Neurol 53 Suppl 3, S3-12; discussion S12-15, doi:10.1002/ana.10513 (2003).

16 Zhai, S., Tanimura, A., Graves, S. M., Shen, W. \& Surmeier, D. J. Striatal synapses, circuits, and Parkinson's disease. Curr Opin Neurobiol 48, 9-16, doi:10.1016/j.conb.2017.08.004 (2018).

17 Isacson, O. Problems and solutions for circuits and synapses in Parkinson's disease. Neuron 43, 165-168, doi:10.1016/j.neuron.2004.07.007 (2004).

18 Martinu, K. \& Monchi, O. Cortico-basal ganglia and cortico-cerebellar circuits in Parkinson's disease: pathophysiology or compensation? Behav Neurosci 127, 222-236, doi:10.1037/a0031226 (2013).

19 Wu, T. et al. Basal ganglia circuits changes in Parkinson's disease patients. Neurosci Lett 524, 55-59, doi:10.1016/j.neulet.2012.07.012 (2012).

20 DeLong, M. R. Primate models of movement disorders of basal ganglia origin. Trends Neurosci 13, 281-285 (1990).

21 Smith, Y., Bevan, M. D., Shink, E. \& Bolam, J. P. Microcircuitry of the direct and indirect pathways of the basal ganglia. Neuroscience 86, 353-387 (1998).

22 Day, M., Wokosin, D., Plotkin, J. L., Tian, X. \& Surmeier, D. J. Differential excitability and modulation of striatal medium spiny neuron dendrites. J Neurosci 28, 11603-11614, doi:10.1523/JNEUROSCI.1840-08.2008 (2008).

23 Lardenoije, R. et al. The epigenetics of aging and neurodegeneration. Progress in Neurobiology 131, 21-64, doi:https://doi.org/10.1016/i.pneurobio.2015.05.002 (2015).

24 Feng, Y., Jankovic, J. \& Wu, Y. C. Epigenetic mechanisms in Parkinson's disease. J Neurol Sci 349, 3-9, doi:10.1016/j.jns.2014.12.017 (2015).

25 Ammal Kaidery, N., Tarannum, S. \& Thomas, B. Epigenetic landscape of Parkinson's disease: emerging role in disease mechanisms and therapeutic modalities. Neurotherapeutics 10, 698-708, doi:10.1007/s13311-013-0211-8 (2013).

26 Habibi, E., Masoudi-Nejad, A., Abdolmaleky, H. M. \& Haggarty, S. J. Emerging roles of epigenetic mechanisms in Parkinson's disease. Funct Integr Genomics 11, 523-537, doi:10.1007/s10142-011-0246-z (2011).

27 Chatterjee, P., Roy, D., Bhattacharyya, M. \& Bandyopadhyay, S. Biological networks in Parkinson's disease: an insight into the epigenetic mechanisms associated with this disease. BMC Genomics 18, 721, doi:10.1186/s12864-017-4098-3 (2017).

28 Holliday, R. Epigenetics: a historical overview. Epigenetics 1, 76-80 (2006).

29 Harrison, I. F. \& Dexter, D. T. Epigenetic targeting of histone deacetylase: therapeutic potential in Parkinson's disease? Pharmacol Ther 140, 34-52, doi:10.1016/j.pharmthera.2013.05.010 (2013). 
30 Sanna, M. D., Guandalini, L., Romanelli, M. N. \& Galeotti, N. The new HDAC1 inhibitor LG325 ameliorates neuropathic pain in a mouse model. Pharmacol Biochem Behav 160, 70-75, doi:10.1016/j.pbb.2017.08.006 (2017). 